\title{
Seyfert galaxies with Swift: giant flares, rapid drops, and other surprises
}

\section{S. Komossa*}

Max-Planck-Institut für Radioastronomie, Auf dem Hügel 69, 53121 Bonn, Germany E-mail:

skomossa@mpifr.de

\section{Grupe}

Department of Earth and Space Science, Morehead State University, 235 Martindale Dr., Morehead, KY 40351, USA

\section{R. Saxton}

XMM SOC, ESAC, Apartado 78, 28691 Villanueva de la Cañada, Madrid, Spain

\section{Gallo}

Department of Astronomy and Physics, Saint Mary's University, 923 Robie Street, Halifax, NS B3H 3C3, Canada

\begin{abstract}
Swift has initiated a new era of understanding the extremes of active galactic nuclei (AGN) variability, their drivers and underlying physics. This is based on its rapid response, high sensitivity, good spatial resolution, and its ability to collect simultaneously X-ray-to-optical SEDs. Here, we present results from our recent monitoring campaigns with Swift of highly variable AGN, including outbursts, deep low states, and unusual long-term trends in several Seyfert galaxies including Mrk 335, WPVS 007, and RXJ2314.9+2243. We also report detection of a new X-ray and optical outburst of IC 3599 and our Swift follow-ups. IC 3599 was previously known as one of the AGN with the highest-amplitude outbursts. We briefly discuss implications of this second outburst of IC 3599 for emission scenarios including accretion-disk variability, repeat tidal disruption events, and the presence of a binary supermassive black hole.
\end{abstract}

Swift: 10 Years of Discovery

2-5 December 2014

La Sapienza University, Rome, Italy

${ }^{*}$ Speaker. 


\section{Introduction}

AGN variability provides us with a powerful tool of understanding the physics of the central engine [1]. While many AGN show mild variability, a few of them exhibit high-amplitude outbursts or low states, changing their flux by factors 20-50, or in very rare cases exceeding factors of 100 . Spectral changes during these extreme states inform us about the processes near the supermassive black hole (SMBH), including reflection and relativistic effects, accretion physics, and the presence and properties of cold and highly ionized absorbers. In order to catch AGN in extreme states, multi-wavelength long-term monitoring, and dedicated follow-ups once a source flares or dips, are required. The Swift mission [2] has provided important contributions to this field. It has served as both: the discovery mission, triggering deep follow-ups with other instruments; or as follow-up mission, providing invaluable information on the lightcurve evolution of peculiar states of sources.

\section{Mrk 335: the AGN that cannot make up its mind}

Mrk 335 is a prominent, nearby, bright Seyfert galaxy, which has been observed with every major previous X-ray mission. Swift discovered a historic minimum flux state [3]. The long-term X-ray lightcurve of Mrk 335 is shown in Fig. 1. While it initially spent most of the time in a higher state, in recent years, it has increasingly often been found in X-ray weak states plus occasional flaring. The 2014 lightcurve of Mrk 335 (Fig. 2) shows a strong flare, which peaked in Sept. 2014 followed by a rapid decline. The peak flux reached a value not seen since 2008 .

The intermediate and low states are characterized by strong spectral complexity. These states were followed-up with deep XMM-Newton and broad-band Suzaku spectroscopy. The XMMNewton observations have revealed the presence of highly ionized absorption [4,5]. Broad-band Suzaku spectroscopy [6] of a recent deep low state of Mrk 335 has shown that it is well explained with a blurred reflection scenario (then requiring high black hole spin), or alternatively with a partial-covering absorber (nearly Compton-thick in low state).

\section{WPVS 007: the AGN that disappeared}

WPVS 007 almost vanished from the X-ray sky $\sim 25$ years ago [7], while its optical spectrum remains that of a narrow-line Seyfert 1 (NLS1) galaxy, and unchanged. UV spectroscopy [8] revealed the likely cause of the X-ray faintness: the onset and development of a strong broadabsorption line (BAL) flow, remarkable for this low-mass galaxy. Swift monitoring [9] discovered occasional flickering into X-ray bright states, presumably due to leaky absorption, and highamplitude UV variability (Fig. 3). The recovery into its "normal state" is yet to come, and will provide us with an excellent opportunity of studying BAL processes on short time scales.

\section{IC 3599: the AGN that re-appeared}

Swift discovery of a new outburst. The Seyfert galaxy IC 3599 (Zwicky 159.034) underwent a dramatic X-ray outburst during the ROSAT all-sky survey by a factor $\sim 100$, accompanied by a strong brightening of the optical emission lines which then faded again $[10,11,12]$. Several outburst 
scenarios were discussed, including high-amplitude NLS1 variability, a disk instability, or a stellar tidal disruption event (TDE). IC 3599 was already known as a Seyfert galaxy before the outburst (based on its narrow emission lines like [OIII]), so it was difficult to make a strong case for a TDE, because of the long-term presence of an accretion disk in that galaxy, and therefore the possibility to link the high-amplitude variability to accretion processes (similar to, but more extreme than, other AGN).

IC 3599 had remained very faint ever since, but was recently discovered in another outburst with Swift (Fig. 4). Inspecting data from the Catalina sky survey [13], we find that the X-ray emission was accompanied by enhanced optical emission, which was already at similar brightness 2 years earlier (Fig. 4). After we noticed the new high state, we triggered new observations with Swift, which show that the 2010 outburst is over, and X-ray emission levels are down again by a factor $\sim 100$. How does this second outburst constrain emission scenarios discussed earlier? Which new ones do emerge? Below, we briefly raise some possibilities. Aspects of these models, and the full data sets, are further discussed by [14].

TDE-related outburst scenarios. At first glance, the detection of repeated flaring is unexpected, if the TDE interpretation was correct. However, we note that recurrent outbursts or high states may also occur in TDE scenarios, for instance when one of the following conditions is met: (1) If IC 3599 hosts a binary black hole or recoiling black hole, tidal disruption rates are temporarily strongly boosted (e.g., $[15,16,17]$ ), and a new disruption event can occur within a decade. (2) If a TDE in IC 3599 happened in a binary SMBH, its lightcurve would show characteristic recurrent dips, since the presence of the secondary temporarily interrupts the accretion stream on the primary [18]; a model successfully applied to the TDE lightcurve from SDSSJ120136.02+300305.5 [19]. (3) Some very loosely bound clumps of stellar debris may return late to the BH causing occasional additional accretion flares, even though it is unlikely that they produce a peak luminosity similar

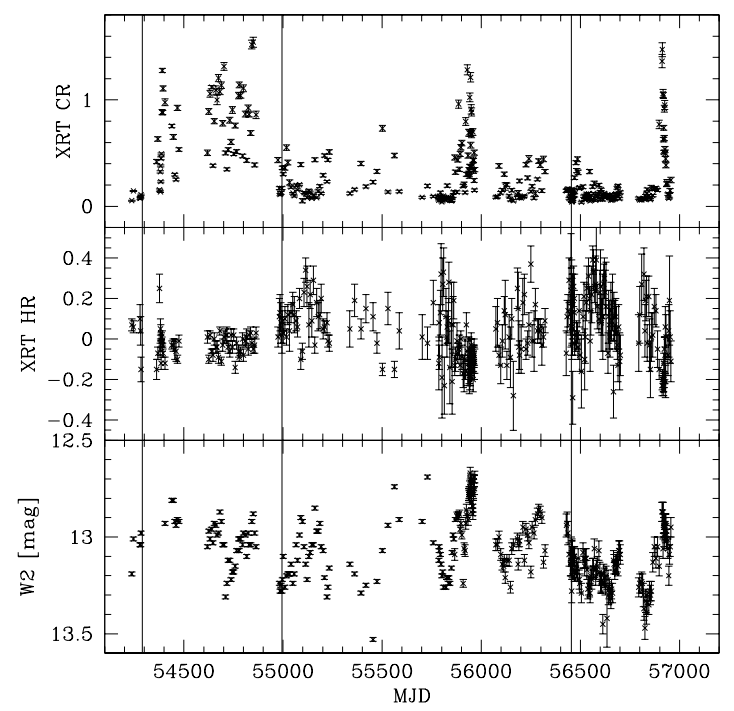

Figure 1: Swift long-term lightcurve of Mrk 335 (upper panel: X-ray telescope (XRT) countrate, middle panel: hardness ratio, lower panel: UVOT W2 magnitude). 


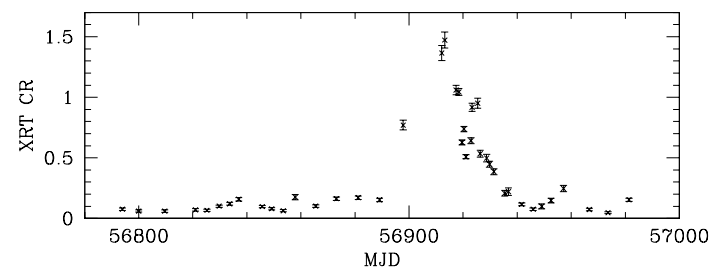

Figure 2: Zoom on the recent flaring activity of Mrk 335 which peaked in September 2014.

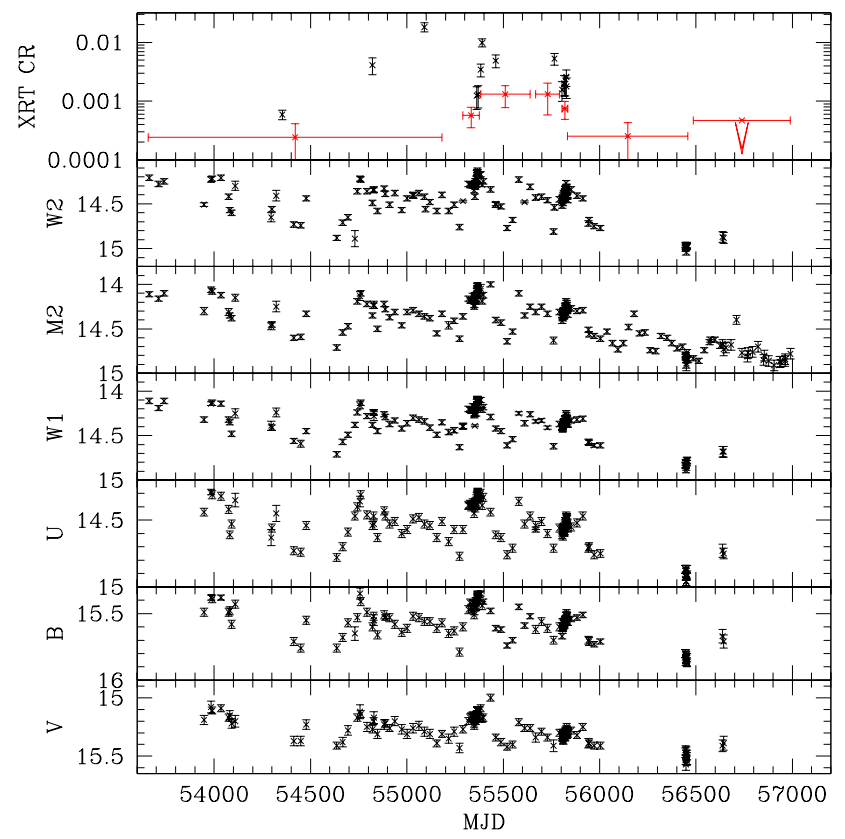

Figure 3: Swift long-term lightcurve of WPVS 007 until December 2014. No more detections in X-rays have occurred during the last year, and the UV shows an overall downward trend, too.

to the earlier one. (4) Another variant of explaining the new lightcurve of IC 3599 with a TDE was presented by [20] at this Swift meeting.

AGN-related outburst scenarios. Given that IC 3599 hosts a long-lived AGN, extreme processes in its accretion disk, possibly due to instabilities, are a possible cause of its recurrent outbursts. We note in passing that an extreme absorption event like the one in WPVS 007 is a very unlikely explanation for the lightcurve of IC 3599, because of the significant brightening of several optical emission lines of IC 3599, strongly arguing for a true outburst at that time. If the outbursts continue repeating, the behaviour is reminiscent of OJ287 (e.g., [21,22]), and a binary SMBH might be responsible (i.e., a secondary $\mathrm{BH}$ interacting with the accretion disk around the primary while orbiting). Another mechanism which produces repeat outbursts is episodic stream-feeding of one of the black holes in a binary SMBH system [23] which recurs every orbit. Inbetween the outbursts, the X-ray emission is weak. Ongoing monitoring with Swift will provide tight new constraints on outburst models. 

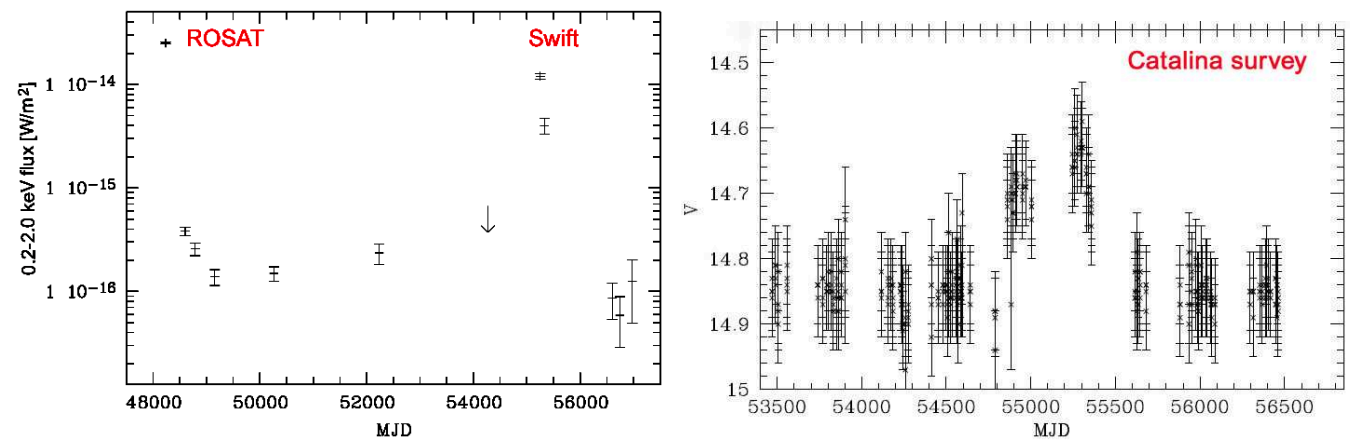

Figure 4: Left: X-ray lightcurve of IC 3599, starting with the initial outburst detected with ROSAT. The last data points are from Swift, which found IC 3599 back in a high state in 2010. We therefore triggered follow-ups with Swift, which showed that flux levels have strongly decreased again. Right: Catalina survey lightcurve of IC 3599, showing that the new X-ray peak was accompanied by enhanced emission in the optical, which was already bright in 2008, and still (or again) in 2010. Increased optical and UV emission was also seen with Swift in 2010.

\section{XMM J061927.1-655311: giant AGN outburst or a TDE?}

XMM J0619-65 was caught in a high-amplitude flaring state (factor $>140$ ) with XMM-Newton $\left(L_{\mathrm{x}, \text { peak }} \sim 10^{44} \mathrm{erg} / \mathrm{s} ; \Gamma_{\mathrm{x}} \simeq 2\right.$ ). Optical follow-up spectroscopy revealed low-level AGN activity. The X-rays and UV, observed with Swift, faded subsequently [24]. Most likely, we have seen an extreme case of AGN variability (a change in accretion rate). Alternatively, a TDE occurred at the core of this galaxy.

\section{RXJ2314.9+2243: an extreme and radio-loud NLS1 galaxy}

RXJ2314.9+2243 is one of the few radio-loud NLS1 galaxies [25], with a tentative $\gamma$-ray detection (L. Foschini, priv.com.; Berton et al. 2015, in prep.). With Swift, we have measured its UV-X-ray spectral energy distribution (SED) quasi-simultaneously for the first time [26]. Its SED shows a broad hump from the IR to UV with a steep decline in the UV, unlike radio-quiet NLS1 galaxies [27], but consistent with a Synchrotron origin of the emission. Overall, RXJ2314.9+2243 shares the dual properties of blazars and NLS1s exhibited by the few other known radio-loud $\gamma$ ray-emitting NLS1 galaxies. Further, it shows a remarkably strong outflow component in its broad [OIII] emission line $(v=1260 \mathrm{~km} / \mathrm{s})$, and likely represents an extreme case of AGN-induced feedback in the local universe [26].

\section{References}

[1] A.C. Fabian, Probing General Relativity with Accreting Black Holes, IAUS 290 (2013) 3

[2] N. Gehrels, et al., The Swift Gamma-Ray Burst Mission, ApJ 611 (2004) 1005

[3] D. Grupe, et al., Discovery of the Narrow-Line Seyfert 1 Galaxy Markarian 335 in a Historical Low $X$-Ray Flux State, ApJ, 668 (2007) L111

[4] D. Grupe, et al., A Remarkable Long-term Light Curve and Deep, Low-state Spectroscopy: Swift and XMM-Newton Monitoring of the NLS1 Galaxy Mkn 335, ApJS, 199 (2012) 28 
[5] A. Longinotti, et al., The Rise of an Ionized Wind in the Narrow-line Seyfert 1 Galaxy Mrk 335 Observed by XMM-Newton and HST, ApJ, 766 (2013) 104

[6] L.C. Gallo, et al., Suzaku observations of Mrk 335: confronting partial covering and relativistic reflection, MNRAS 446 (2015) 633

[7] D. Grupe, et al., Discovery of an ultrasoft transient ROSAT AGN: WPVS 007, Astron. Astrophys. 300 (1995) L21

[8] K.M. Leighly, et al., Emergence of a Broad Absorption Line Outflow in the Narrow-line Seyfert 1 Galaxy WPVS 007, ApJ 701 (2009) 176

[9] D. Grupe, et al., Strong UV and X-Ray Variability of the Narrow Line Seyfert 1 Galaxy WPVS 007-on the Nature of the X-Ray Low State, AJ 146 (2013) 78

[10] W.N. Brandt, et al., The unusual X-ray and optical properties of the ultrasoft active galactic nucleus Zwicky 159.034 (RE J1237+264), MNRAS 273 (1995) L47

[11] D. Grupe, et al., X-ray outburst of the peculiar Seyfert galaxy IC 3599, Astron. Astrophys. 299 (1995) L5

[12] S. Komossa, N. Bade, The giant X-ray outbursts in NGC 5905 and IC 3599: Follow-up observations and outburst scenarios, Astron. Astrophys. 343 (1999) 775

[13] A.J. Drake, et al., First Results from the Catalina Real-Time Transient Survey, ApJ 696 (2009) 870

[14] D. Grupe, et al., IC 3599 Did It Again: A Second Outburst of the X-Ray Transient Seyfert 1.9 Galaxy, ApJ 803 (2015) L28

[15] X. Chen, et al., Enhanced Tidal Disruption Rates from Massive Black Hole Binaries, ApJ 697 (2009) L149

[16] S. Komossa, D. Merritt, Tidal Disruption Flares from Recoiling Supermassive Black Holes, ApJ 683 (2008) L21

[17] N. Stone, A. Loeb, Prompt tidal disruption of stars as an electromagnetic signature of supermassive black hole coalescence, MNRAS 412 (2011) 75

[18] F.K. Liu, et al., Interruption of Tidal-Disruption Flares by Supermassive Black Hole Binaries, ApJ 706 (2009) L133

[19] F.K. Liu, et al., A Milliparsec Supermassive Black Hole Binary Candidate in the Galaxy SDSSJ120136.02+300305.5, ApJ 786 (2014) 103

[20] D. Mainetti, et al., these proceedings, (2015)

[21] A. Sillanpää, et al., OJ 287 - Binary pair of supermassive black holes, ApJ 325 (1988) 628

[22] M. Valtonen, et al., A massive binary black-hole system in $0 J 287$ and a test of general relativity, Nature 452 (2008) 851

[23] T.L. Tanaka, Recurring flares from supermassive black hole binaries: implications for tidal disruption candidates and OJ 287, MNRAS 434 (2013) 2275

[24] R.D. Saxton, et al., An X-ray and UV flare from the galaxy XMMSL1 J061927.1-655311, Astron. Astrophys. 572 (2014) 1

[25] S. Komossa, et al., Radio-loud Narrow-Line Type 1 Quasars, AJ 132 (2006) 531 
[26] S. Komossa, et al., What powers the radio-loud narrow-line Seyfert 1 galaxy RX J2314.9+2243?. A view onto its central engine from radio to X-rays, Astron. Astrophys. 574 (2015) 121

[27] D. Grupe, et al., The Simultaneous Optical-to-X-Ray Spectral Energy Distribution of Soft X-Ray Selected Active Galactic Nuclei Observed by Swift, ApJS 187 (2010) 64 